ANNALES

POLONICI MATHEMATICI

$80(2003)$

\title{
On roots of polynomials with power series coefficients
}

\author{
by RafaŁ PierzchaŁa (Kraków)
}

\begin{abstract}
We give a deepened version of a lemma of Gabrielov and then use it to prove the following fact: if $h \in \mathbb{K}[[X]](\mathbb{K}=\mathbb{R}$ or $\mathbb{C})$ is a root of a non-zero polynomial with convergent power series coefficients, then $h$ is convergent.
\end{abstract}

This article is inspired by Lemma 1.2 of Gabrielov [2]. Roughly speaking, it states the following: if $A$ is an integral domain, $P(Z, T)=T^{p}+$ $a_{1}(Z) T^{p-1}+\ldots+a_{p}(Z) \in A[[Z]][T], f=\sum_{\nu=0}^{\infty} f_{\nu} Z^{\nu} \in A[[Z]]$ is a formal power series for which $P(Z, f(Z))=0$ and $(\partial P / \partial T)(Z, f(Z)) \neq 0$, then there exists a non-negative integer $\nu_{0}$ such that for $\nu \geq \nu_{0}, f_{\nu}$ has a polynomial expresion in the coefficients of $a_{i}, 1 \leq i \leq p$, and $f_{k}, k \leq \nu_{0}$, and some constant $g \in A$ depending on $f$ and $P$. Moreover some good estimates hold for the degrees of these polynomials. However, nothing is said about estimates of the coefficients, except that they are integers, and unfortunately no reasonable conclusions about these coefficients can be easily derived from the proof. That is the reason why we formulate and prove a deepened version of Gabrielov's result (Theorem 1).

Throughout this paper $\mathbb{K}$ denotes either the field $\mathbb{R}$ of real numbers or the field $\mathbb{C}$ of complex numbers. Fix $m \in \mathbb{N}$. We will denote $\left(X_{1}, \ldots, X_{m}\right)$ by $X$, and $Y, Z, T$ will always signify single indeterminates. Take a multiindex $\alpha=\left(\alpha_{1}, \ldots, \alpha_{m}\right) \in \mathbb{N}^{m}$. We will write $X^{\alpha}$ instead of the formal monomial $X_{1}^{\alpha_{1}} \ldots X_{m}^{\alpha_{m}}$. Moreover $|\alpha|$ signifies the sum $\alpha_{1}+\ldots+\alpha_{m}$. In $\mathbb{K}^{m}$ we will consider the Euclidean norm $\|\cdot\|$. Recall that a formal power series $\sum_{\alpha \in \mathbb{N}^{m}} a_{\alpha} X^{\alpha}, a_{\alpha} \in \mathbb{K}$, is called convergent if it is convergent in some neighbourhood of the origin. This is the case if and only if there are some constants $M, R>0$ such that $\left|a_{\alpha}\right| \leq M R^{|\alpha|}$. The ring of convergent power series will be denoted by $\mathbb{K}\{X\}$. Finally, for $p \in \mathbb{N}$ we put $I_{p}:=\{n \in \mathbb{N}$ : $n \leq p\}$.

2000 Mathematics Subject Classification: Primary 13F25; Secondary 32B20, 16W60.

Key words and phrases: formal power series, convergent power series, Newton-Puiseux theorem. 
TheOREM 1. Let $A$ be an integral domain and $p$ a positive integer. Suppose $P(Z, T)=\sum_{j=0}^{p} c_{p-j}(Z) T^{j} \in A[[Z]][T]$ is a polynomial of degree $p$, $c_{j}(Z)=\sum_{i=0}^{\infty} c_{i, j} Z^{i}, j \in I_{p}$. Let $f(Z)=\sum_{\nu=0}^{\infty} f_{\nu} Z^{\nu} \in A[[Z]]$ be a formal series such that $P(Z, f(Z))=0$ and $(\partial P / \partial T)(Z, f(Z)) \neq 0\left({ }^{1}\right)$. Put $(\partial P / \partial T)(Z, f(Z))=\sum_{l=0}^{\infty} g_{l} Z^{l}$ and let $l_{0}:=\inf \left\{l: g_{l} \neq 0\right\}, g:=-g_{l_{0}}$. Then for each $l>l_{0}$ we have

$$
g^{2\left(l-l_{0}\right)-1} f_{l}=G_{l}\left[\left\{c_{i, j}\right\}\left(i \in I_{l+l_{0}}, j \in I_{p}\right),\left\{f_{\nu}\right\}\left(\nu \in I_{l_{0}}\right), g\right],
$$

where $G_{l}$ is a polynomial with non-negative integral coefficients such that

(i) the degree of $G_{l}$ with respect to $g$ is not greater than $2\left(l-l_{0}\right)-2$;

(ii) the degree of $G_{l}$ with respect to the group of indeterminates $f_{\nu}$ is not greater than $2 p\left(l-l_{0}\right)-p$;

(iii) if we introduce a new indeterminate $Y$ and replace each $c_{i, j}$ by $Y^{i}$, then the degree of $G_{l}$ with respect to $Y$ is not greater than $\left(2 l_{0}+1\right)\left(l-l_{0}\right)$;

(iv) there exists a constant $R>0$ such that the sum of the coefficients of $G_{l}$ is not greater than $R^{l}$.

Proof. The proof of (i)-(iii) is exactly as in [2]. We recall it for the convenience of the reader.

The equality $P(Z, f(Z))=0$ implies that for each $l \in \mathbb{N}$,

$$
c_{l, p}+\sum_{j=0}^{l} \sum_{s=1}^{p} \sum_{\nu_{1}+\ldots+\nu_{s}=j} f_{\nu_{1}} \ldots f_{\nu_{s}} c_{l-j, p-s}=0 .
$$

Take $l>l_{0}$. We have

$$
c_{l+l_{0}, p}+\sum_{j=0}^{l+l_{0}} \sum_{s=1}^{p} \sum_{\nu_{1}+\ldots+\nu_{s}=j} f_{\nu_{1}} \ldots f_{\nu_{s}} c_{l+l_{0}-j, p-s}=0 .
$$

A simple calculation gives

$$
c_{l+l_{0}, p}+\sum_{j=0}^{l+l_{0}} \sum_{s=1}^{p} \sum_{\substack{\nu_{1}+\ldots+\nu_{s}=j \\ \nu_{1}, \ldots, \nu_{s} \leq l-1}} f_{\nu_{1}} \ldots f_{\nu_{s}} c_{l+l_{0}-j, p-s}+\sum_{i=0}^{l_{0}} g_{i} f_{l+l_{0}-i}=0 .
$$

Hence $g^{2\left(l-l_{0}\right)-1} f_{l}$ is equal to

$$
g^{2\left(l-l_{0}\right)-2} c_{l+l_{0}, p}+\sum_{j=0}^{l+l_{0}} \sum_{s=1}^{p} \sum_{\substack{\nu_{1}+\ldots+\nu_{s}=j \\ \nu_{1}, \ldots, \nu_{s} \leq l-1}} g^{2\left(l-l_{0}\right)-2} f_{\nu_{1}} \ldots f_{\nu_{s}} c_{l+l_{0}-j, p-s} .
$$

$\left({ }^{1}\right)$ By the classical Newton-Puiseux theorem (cf. [1]) all roots of the polynomial $P$ are of the form $\left(1 / Z^{m / n}\right) u\left(Z^{1 / n}\right)$, where $m, n \in \mathbb{N}, n>0$ and $u(Z)=\sum_{\nu=0}^{\infty} u_{\nu} Z^{\nu} \in B[[Z]]$ is a power series with coefficients in some integral domain $B \supset A$. 
We put

$$
G_{l_{0}+1}:=c_{2 l_{0}+1, p}+\sum_{j=0}^{2 l_{0}+1} \sum_{s=1}^{p} \sum_{\substack{\nu_{1}+\ldots+\nu_{s}=j \\ \nu_{1}, \ldots, \nu_{s} \leq l_{0}}} f_{\nu_{1}} \ldots f_{\nu_{s}} c_{2 l_{0}+1-j, p-s} .
$$

Suppose that we have defined $G_{k}$ for each $k$ such that $l_{0}+1 \leq k \leq l-1$. We obtain $G_{l}$ by replacing in (1) each $g^{2\left(\nu_{i}-l_{0}\right)-1} f_{\nu_{i}}$ with $G_{\nu_{i}}$ whenever $l_{0}+1 \leq \nu_{i} \leq l-1$. One only needs to note that for $r \in I_{p} \backslash\{0\}$ we have

$$
2\left(l-l_{0}\right)-2 \geq \sum_{i=1}^{r}\left[2\left(\nu_{i}-l_{0}\right)-1\right]
$$

where $\nu_{1}+\ldots+\nu_{r} \leq l+l_{0}$ and $l_{0}+1 \leq \nu_{i} \leq l-1, i \in I_{r} \backslash\{0\}$.

Now we will prove (i)-(iii) by induction on $l \geq l_{0}+1$. The case $l=l_{0}+1$ is clear. Suppose that $l>l_{0}+1$ and the given estimates of the degrees of $G_{k}$ are true for $l_{0}+1 \leq k \leq l-1$. Let $Q$ denote one of the summands of the sum in (1).

CAsE 1: $Q=g^{2\left(l-l_{0}\right)-2} c_{l+l_{0}, p}$. Clearly, the polynomial $Q$ satisfies the degree estimates in (i)-(iii).

CASE 2: $Q=g^{2\left(l-l_{0}\right)-2} f_{\nu_{1}} \ldots f_{\nu_{s}} c_{l+l_{0}-j, p-s}$, where $\nu_{1}+\ldots+\nu_{s}=j$, $\nu_{1}, \ldots, \nu_{s} \leq l_{0}, s \in I_{p} \backslash\{0\}, j \in I_{l+l_{0}}$. In this case the estimates are trivial as well.

CASE 3: $Q=g^{2\left(l-l_{0}\right)-2} f_{\nu_{1}} \ldots f_{\nu_{s}} c_{l+l_{0}-j, p-s}$, where $\nu_{1}+\ldots+\nu_{s}=j$, $\nu_{1} \ldots, \nu_{s} \leq l-1, s \in I_{p} \backslash\{0\}, j \in I_{l+l_{0}}$ and $\nu_{i} \leq l_{0}$ for exactly $s-r$ indices $i \in I_{s} \backslash\{0\}$, where $r \in I_{s} \backslash\{0\}$. We may assume that $\nu_{i} \leq l_{0}$ for $i \geq r+1$. Note that $Q=g^{\alpha} G_{\nu_{1}} \ldots G_{\nu_{r}} Q^{\prime}$, where $Q^{\prime}=f_{r+1} \ldots f_{s} c_{l+l_{0}-j, p-s}, \alpha=$ $2\left(l-l_{0}\right)-2-\sum_{i=1}^{r}\left[2\left(\nu_{i}-l_{0}\right)-1\right]$. As noticed before, $\alpha \geq 0$. Now we apply the induction hypothesis to the polynomials $G_{\nu_{1}}, \ldots, G_{\nu_{r}}$ and easily obtain the required estimates for $Q$.

Now we will prove (iv). Denote by $b_{l}$ the sum of the coefficients of the polynomial $G_{l}$, where $l \geq l_{0}+1$. Put additionally $b_{i}:=1$ for $i \in I_{l_{0}}$. By (1) we get

$$
b_{l}=1+\sum_{j=0}^{l+l_{0}} \sum_{s=1}^{p} \sum_{\substack{\nu_{1}+\ldots+\nu_{s}=j \\ \nu_{1}, \ldots, \nu_{s} \leq l-1}} b_{\nu_{1}} \ldots b_{\nu_{s}}
$$

whenever $l \geq l_{0}+1$. Note that for $l \geq l_{0}+2$,

$$
b_{l}=a b_{l-1}+\sum_{s=1}^{p} \sum_{\substack{\nu_{1}+\ldots+\nu_{s}=l+l_{0} \\ \nu_{1}, \ldots, \nu_{s} \leq l-1}} b_{\nu_{1}} \ldots b_{\nu_{s}},
$$


where $a$ is some constant. One easily verifies that

$$
b_{l}-a b_{l-1}+\sum_{i=0}^{l_{0}} a_{i} b_{l+l_{0}-i}=\sum_{s=1}^{p} \sum_{\nu_{1}+\ldots+\nu_{s}=l+l_{0}} b_{\nu_{1}} \ldots b_{\nu_{s}},
$$

where $a_{0}=\sum_{s=0}^{p-1}(s+1) b_{0}^{s}$, and for $i \geq 1$,

$$
a_{i}=\sum_{s=1}^{p-1} \sum_{\nu_{1}+\ldots+\nu_{s}=i}(s+1) b_{\nu_{1}} \ldots b_{\nu_{s}} .
$$

Let $f$ denote the formal power series $\sum_{j=0}^{\infty} b_{j+l_{0}+1} Z^{j}$ and let $F:=$ $\sum_{i=0}^{l_{0}} b_{i} Z^{i}$. Define also polynomials $H(Z)$ and $G(Z, T)$ as follows:

$$
\begin{aligned}
H(Z) & :=\sum_{j=0}^{2 l_{0}+1} \sum_{s=1}^{p} \sum_{\nu_{1}+\ldots+\nu_{s}=j} b_{\nu_{1}} \ldots b_{\nu_{s}} Z^{j}-\sum_{i+j \leq l_{0}} a_{i} b_{j+l_{0}+1} Z^{i+j+l_{0}+1}, \\
G(Z, T) & :=\left(T-b_{l_{0}+1}\right) Z^{2 l_{0}+1}-a T Z^{2 l_{0}+2}+\sum_{i=0}^{l_{0}} a_{i} T Z^{i+l_{0}+1} .
\end{aligned}
$$

Then

$$
G(Z, f(Z))-\sum_{j=1}^{p}\left[F(Z)+f Z^{l_{0}+1}\right]^{j}+H(Z)=0 .
$$

Note first that $f$ is the only formal series which satisfies the identity above. One checks next that

$$
\sum_{j=1}^{p}\left[F(Z)+T Z^{l_{0}+1}\right]^{j}=\sum_{i=0}^{l_{0}} a_{i} T Z^{i+l_{0}+1}+H(Z)+Z^{2 l_{0}+2} P(Z, T),
$$

where $P(Z, T)$ is a polynomial. Hence $W(Z, f(Z))=0$, where

$$
W(Z, T):=T-b_{l_{0}+1}-a T Z-P(Z, T) Z .
$$

Since $W\left(0, b_{l_{0}+1}\right)=0$ and $(\partial W / \partial T)\left(0, b_{l_{0}+1}\right) \neq 0$, it follows that there is a convergent power series $h$ such that $W(Z, h(Z))=0$. On the other hand, as mentioned before, the coefficients of $h$ are uniquely determined, so $f=h$ as formal power series. Therefore $f$ is convergent and there is a positive constant $R$ such that $b_{l} \leq R^{l}$ for $l \geq l_{0}+1$. The proof of the theorem is now complete.

REMARK 1. It follows from the proof that $g$ is a polynomial in $c_{i, j}$ and $f_{\nu}\left(i \in I_{l_{0}}, j \in I_{p}, \nu \in I_{l_{0}}\right)$ with integral coefficients.

Lemma 1. Consider a formal series $A=\sum_{n=0}^{\infty} A_{n} Y^{n} \in \mathbb{K}[[X]][[Y]]$. Then $A$ is convergent if and only if there are constants $r, R, M>0$ such that the series $A_{n}$ is convergent in the open ball $\{\|x\|<2 r\}$ and $\sup \left\{\left|A_{n}(x)\right|\right.$ : $\|x\| \leq r\} \leq M R^{n}$ for each $n \in \mathbb{N}$. 
Proof. Write $A_{n}=\sum_{\alpha \in \mathbb{N}^{m}} A_{\alpha}^{(n)} X^{\alpha}, A_{\alpha}^{(n)} \in \mathbb{K}$, and suppose $A$ is convergent. There are positive constants $C, R$ such that $\left|A_{\alpha}^{(n)}\right| \leq C R^{|\alpha|+n}$. Take $r:=(4 R)^{-1}$. Clearly, each $A_{n}$ is convergent in $\{\|x\|<2 r\}$ and for $\|x\| \leq r$ we have $\left|A_{n}(x)\right| \leq \sum_{\alpha \in \mathbb{N}^{m}} C R^{n} 4^{-|\alpha|}=M R^{n}$. The reverse implication follows from the Weierstrass criterion of convergence.

For $m \geq 2$ let $\varphi_{m}$ denote the following map:

$$
\varphi_{m}: \mathbb{K}^{m} \ni\left(x_{1}, \ldots, x_{m}\right) \mapsto\left(x_{1} x_{m}, \ldots, x_{m-1} x_{m}, x_{m}\right) \in \mathbb{K}^{m} .
$$

Lemma 2. A power series $A \in \mathbb{K}[[X, Y]]$ is convergent if and only if $A\left(\varphi_{m}(X), Y\right)$ is convergent.

Proof. Suppose the power series $A\left(\varphi_{m}(X), Y\right)$ is convergent. Since

$$
A\left(\varphi_{m}(X), Y\right)=\sum_{\alpha \in \mathbb{N}^{m+1}} a_{\alpha} X_{1}^{\alpha_{1}} \ldots X_{m}^{\alpha_{m}} X_{m}^{\alpha_{1}+\ldots+\alpha_{m-1}} Y^{\alpha_{m+1}},
$$

where $a_{\alpha} \in \mathbb{K}$ are the coefficients of $A$, then there are positive constants $M, R$ such that $\left|a_{\alpha}\right| \leq M R^{|\alpha|+\alpha_{1}+\ldots+\alpha_{m-1}}$. We may assume that $R>1$ and then $\left|a_{\alpha}\right| \leq M R^{2|\alpha|}$. This implies the convergence of $A$. The reverse implication is trivial.

Lemma 3. Suppose that a non-zero formal power series $G \in \mathbb{K}[[X]]$ and a formal power series $H(X, Y)=\sum_{n=0}^{\infty} F_{n}(X) G^{n}(X) Y^{n}$ are convergent, where $F_{n}(X) \in \mathbb{K}[[X]]$. Then $F(X, Y)=\sum_{n=0}^{\infty} F_{n}(X) Y^{n}$ is convergent as well.

Proof. We may assume that $m \geq 2$, because any power series in one variable can be treated as a power series in two variables. Write $G=\sum_{\nu=\nu_{0}}^{\infty} G_{\nu}$ as the sum of homogeneous polynomials of degree $\nu$, where $G_{\nu_{0}} \neq 0$. Take $a \in \mathbb{K}^{m} \backslash\{0\}$ such that $G_{\nu_{0}}(a) \neq 0$. There is a linear automorphism $L: \mathbb{K}^{m} \rightarrow \mathbb{K}^{m}$ such that $L((0,0, \ldots, 1))=a$. Then

$$
\left(G \circ L \circ \varphi_{m}\right)(X)=\sum_{\nu=\nu_{0}}^{\infty} G_{\nu}\left(L\left(X_{1}, \ldots, X_{m-1}, 1\right)\right) X_{m}^{\nu}=X_{m}^{\nu_{0}} P(X),
$$

where $P(X)$ is an invertible convergent power series. Since $L$ is an automorphism, it is enough to show that $F\left(L\left(\varphi_{m}(X)\right), Y\right.$ ) is convergent (cf. Lemma 2). Write $F_{n}\left(L\left(\varphi_{m}(X)\right)=\sum_{\alpha \in \mathbb{N}^{m}} b_{\alpha}^{(n)} X^{\alpha}, b_{\alpha}^{(n)} \in \mathbb{K}\right.$. We need to show that there are some constants $M, R>0$ such that $\left|b_{\alpha}^{(n)}\right| \leq M R^{|\alpha|+n}$, where $\alpha \in \mathbb{N}^{m}, n \in \mathbb{N}$. Clearly, the formal power series

$$
H\left(L\left(\varphi_{m}(X)\right), P^{-1}(X) Y\right)=\sum_{n=0}^{\infty}\left[\sum_{\alpha \in \mathbb{N}^{m}} b_{\alpha}^{(n)} X^{\alpha}\right] X_{m}^{\nu_{0} n} Y^{n}
$$

is convergent. Therefore there are positive constants $M, C$ such that $\left|b_{\alpha}^{(n)}\right| \leq$ $M C^{|\alpha|+\nu_{0} n+n}$. We may assume that $C \geq 1$ and then $\left|b_{\alpha}^{(n)}\right| \leq M R^{|\alpha|+n}$, where $R:=C^{\nu_{0}+1}$. 
We have the following consequence of Lemma 3:

Corollary. Suppose $A(X) B(X)=C(X)$, where $A, B, C \in \mathbb{K}[[X]]$ and $B, C$ are convergent. Then $A$ is convergent.

Proof. We apply Lemma 3 to $H(X, Y)=A(X) B(X) Y, G(X)=B(X)$ and $F(X, Y)=A(X) Y$.

Though we will not apply this corollary in our paper, we have stated it to show that the idea of using the map $\varphi_{m}$ gives a very short and elementary proof of it.

Now we will give a new elementary proof of a certain theorem, proved in [3] as a consequence of Artin's Approximation Theorem:

Theorem 2. Let $m$ and $p$ be positive integers. Suppose that $S(X, T)=$ $\sum_{j=0}^{p} a_{p-j}(X) T^{j} \in \mathbb{K}\{X\}[T]$ is a non-zero polynomial. Assume as well that $h \in \mathbb{K}[[X]]$ satisfies the identity $S(X, h(X))=0$. Then $h$ is convergent.

Proof. We may assume that $S$ is of the lowest possible degree. Hence $(\partial S / \partial T)(X, h(X)) \neq 0$. Moreover we may assume that $m \geq 2$. Write $h=\sum_{\nu=0}^{\infty} h_{\nu}$ as the sum of homogeneous polynomials $h_{\nu}$ of degree $\nu$. It follows from Lemma 2 that it suffices to prove that the power series $f=h \circ \varphi_{m}$ is convergent. Put $f_{\nu}\left(X_{1}, \ldots, X_{m-1}\right):=h_{\nu}\left(\varphi_{m}\left(X_{1}, \ldots, X_{m-1}, 1\right)\right)$. Obviously, $f(X)=\sum_{\nu=0}^{\infty} f_{\nu}\left(X_{1}, \ldots, X_{m-1}\right) X_{m}^{\nu}$. Let $P(X, T):=S\left(\varphi_{m}(X), T\right)$ and $c_{j}:=a_{j} \circ \varphi_{m}$ for $j \in I_{p}$. Clearly, $P(X, f(X))=0$ and $(\partial P / \partial T)(X, f(X))$ $\neq 0$. Write $c_{j}(X)=\sum_{i=0}^{\infty} c_{i, j}\left(X_{1}, \ldots, X_{m-1}\right) X_{m}^{i}$. There is a neighbourhood of the origin in $\mathbb{K}^{m-1}$ in which each $c_{i, j}$ is convergent; we may assume that $\left|c_{0, j}(x)\right| \leq 1$ for each $x$ in that neighbourhood. Now we apply Theorem 1 to $f$ and the polynomial $P$ putting $A:=\mathbb{K}\left[\left[X_{1}, \ldots, X_{m-1}\right]\right]$. We obtain a convergent power series $g$ (cf. Remark 1 ), an integer $l_{0}$ and polynomials $G_{l}, l \geq l_{0}+1$. It follows from Theorem 1 and Lemma 1 that $\sum_{l=l_{0}+1}^{\infty} g^{2\left(l-l_{0}\right)-1} f_{l} X_{m}^{l}$ is convergent, and so is the formal power series $\sum_{l=l_{0}+1}^{\infty} f_{l} g^{2 l} X_{m}^{l}$. Therefore by Lemma $3, f$ is convergent.

Acknowledgements. I would like to thank Professor Wiesław Pawłucki for suggesting the problem and for his help during the preparation of this paper. I am also indebted to my friend Marcin Skrzyński who helped me to correct the text.

\section{References}

[1] E. Brieskorn and H. Knörrer, Ebene algebraische Kurven, Birkhäuser, Basel, 1981.

[2] A. M. Gabrielov, Formal relations among analytic functions, Izv. Akad. Nauk SSSR 37 (1973), 1056-1090 (in Russian). 
[3] J. M. Ruiz, The Basic Theory of Power Series, Adv. Lectures in Math., Vieweg, Braunschweig, 1993.

Institute of Mathematics

Jagiellonian University

Reymonta 4

30-059 Kraków, Poland

E-mail: pierzcha@im.uj.edu.pl 